\title{
Recomendaciones para el diagnóstico y el tratamiento de los síntomas posquirúrgicos persistentes en la enfermedad de Hirschsprung
}

\author{
Recommendations for the diagnosis and treatment of persistent postsurgical \\ symptoms in Hirschsprung disease
}

\author{
Dra. Ana M. Rocca ${ }^{a}$,Dra. Mariana Nastric, Dra. Silvia Takedab , Dra. Daniela Neder ${ }^{a}$, \\ Dra. Alejandra Mortarini ${ }^{a}$ Dr. Enrique Paz ${ }^{b}$, Lic. Silvana Lavorgnad ${ }^{d}$ Lic. Mariana Bazo $0^{d} y$ \\ Dr. Víctor Dibenedetto ${ }^{b}$
}

\section{RESUMEN}

La enfermedad de Hirschsprung ocurre en 1 de cada 5000 nacimientos. La falla de migración de las células ganglionares desde la cresta neural en dirección cefalocaudal genera su ausencia en parte o todo el colon. Se manifiesta con falta de eliminación de meconio, distensión abdominal y dificultades en la evacuación. Luego del tratamiento quirúrgico, existen complicaciones a corto y largo plazo. El objetivo de esta publicación es describir las principales causas de síntomas persistentes en los pacientes operados por enfermedad de Hirschsprung y presentar un algoritmo diagnóstico-terapéutico factible de ser realizado en nuestro medio.

Palabras clave: enfermedad de Hirschsprung, enterocolitis, estreñimiento, incontinencia fecal.

\section{ABSTRACT \\ Hirschsprung disease is characterized by the lack of migration of intrinsic parasympathetic ganglia from neural crest and consequently absence of them at varying length of the bowel, resulting in functional obstruction. The incidence is 1 per 5000 births. After surgery, short term and long term comorbidity commonly occurs. The aim of this article is to revise the main causes of ongoing symptoms after surgery in Hirschsprung disease patients and to show a diagnostic and therapeutic algorithm that can be developed in our community. \\ Key words: Hirschsprung disease, enterocolitis, constipation, fecal incontinence. \\ http: / / dx.doi.org/ 10.5546/ aap.2020.350}

Cómo citar: Rocca AM, Nastri M, Takeda S, Neder D, et al. Recomendaciones para el diagnóstico y el tratamiento de los síntomas posquirúrgicos persistentes en la enfermedad de Hirschsprung. Arch Argent Pediatr 2020;118(5):350-357.

a. Servicio de Gastroenterología.

b. Servicio de Cirugía.

c. Servicio de Pediatría.

d. Área de Alimentación.

Hospital de Pediatría SAMIC "Prof. Dr. Juan P. Garrahan",

Ciudad Autónoma de Buenos Aires, Argentina.

Correspondencia:

Dra. Ana M. Rocca: anmarocca@gmail.com

Financiamiento: Ninguno.

Conflicto de intereses: Ninguno que declarar.

Recibido: 6-12-2019

Aceptado: 6-5-2020

Publicado Primero en Internet: 1-9-2020

\section{INTRODUCCIÓN}

La enfermedad de Hirschsprung (EH) ocurre en 1 de cada 5000 nacimientos. Se debe a la ausencia de células ganglionares en los plexos mientérico y submucoso de parte o todo el colon por una falla en la migración desde la cresta neural. Se manifiesta con falta de eliminación de meconio, obstrucción intestinal en el período neonatal, distensión abdominal y constipación. En la mayoría, se diagnostica antes de los 6 meses, pero está descrita en niños mayores y adultos. ${ }^{1}$ Los estudios iniciales incluyen colon por enema y manometría anorrectal. Se confirma con biopsia rectal, que demuestra la ausencia de células ganglionares.

La cirugía correctora de la EH ha evolucionado notablemente en las últimas décadas. Las primeras técnicas realizaban una disección transmural (Swenson), ${ }^{2}$ un descenso endorrectal $(\text { Soave })^{3}$ o retrorrectal (Duhamel) ${ }^{4}$ que dejaban ostomía. Luego, se incorporó el descenso primario más precoz, por vía transanal exclusiva o combinada con abordaje laparoscópico (técnica de Georgeson). ${ }^{5}$ En nuestro Centro, se realiza la operación de Georgeson en los primeros meses de vida, sin colostomía previa siempre que se pueda manejar adecuadamente al paciente con irrigaciones colónicas.

Pese a una técnica quirúrgica adecuada, el $50 \%$ de los pacientes no tienen continencia o hábito defecatorio normales o tienen una combinación de problemas. ${ }^{6}$ El antiguo paradigma de que estos síntomas mejoran con la edad se ha modificado. ${ }^{7}$ Un estudio finlandés realizado en adultos con $\mathrm{EH}$ encontró que solo el $47 \%$ tenía un puntaje de continencia normal. El $48 \%$ experimentaba episodios de incontinencia ocasional, socialmente incapacitante en un $14 \% .{ }^{8}$ Esto llevó a crear diversos algoritmos para el diagnóstico y el tratamiento para intentar mejorar la continencia y la calidad de vida. ${ }^{9-11}$ 
Al ser una patología de resolución inicialmente quirúrgica, se requiere la intervención de pediatras, gastroenterólogos y nutricionistas para el diagnóstico y el tratamiento de los síntomas persistentes. En el Hospital Garrahan, se creó un consultorio multidisciplinario a fin de optimizar la atención y favorecer la discusión de casos complejos. El objetivo de esta publicación es revisar las principales causas de síntomas persistentes y mostrar un algoritmo diagnósticoterapéutico aplicable en nuestro medio.

\section{COMPLICACIONES POSOPERATORIAS SÍNTOMAS OBSTRUCTIVOS}

Se manifiestan con distensión abdominal, borborigmos, vómitos, constipación. ${ }^{8}$

\section{Causas:}

1. Obstrucción mecánica: estenosis de la anastomosis, compresión por la bolsa aganglionar (cirugía de Duhamel) o torsión del descenso. Se diagnostica por tacto rectal y colon por enema con contraste hidrosoluble. Los cuadros suboclusivos debidos a bridas pueden evidenciarse por radiografía de abdomen simple o estudio contrastado del intestino delgado.

2. Aganglionosis residual: por error de anatomía patológica o descenso del segmento de transición. Se confirma por biopsia rectal por succión.

3. Alteraciones de la motilidad: en el colon remanente ganglionar o en el intestino delgado como consecuencia de una afectación más generalizada del sistema nervioso entérico (SNE). ${ }^{6}$ Se realiza manometría anorrectal, con sedación en los pacientes pequeños..$^{12,13}$ Si es normal, se estudia el intestino proximal con manometría colónica ${ }^{13} \mathrm{o}$ con tránsito con marcadores radiopacos, y se evalúa con radiografía de abdomen el porcentaje de anillos eliminados al $5^{\text {to }}$ día (normal: el $80 \%$ ) y la distribución de los remanentes. ${ }^{14}$

4. Acalasia del esfínter o esfínter anal interno (EAI) hipertensivo: presente en un $5 \%$ de los pacientes. La manometría muestra presiones de esfínter interno mayores de $50 \mathrm{mmHg}$. El tratamiento consiste en la inyección de toxina botulínica a nivel de la línea pectínea, en dosis de $6 \mathrm{U} / \mathrm{kg}$ (hasta $100 \mathrm{U}$ ) divididas en los cuatro cuadrantes. ${ }^{15}$ Es un procedimiento poco invasivo y con menor riesgo de incontinencia a largo plazo; en muchos centros, ha reemplazado a miotomías o miectomías. ${ }^{6,9,11,16}$
5. Constipación funcional: por desarrollo de conductas retentivas luego de la cirugía en relación con situaciones que generan dolor, como dermatitis perianal, dilataciones, enemas. ${ }^{5}$ La manometría anorrectal, que no es obligatoria, muestra la falta de relajación del esfínter anal externo durante el pujo o comportamiento paradojal. El tratamiento es el de la constipación funcional.

\section{Conducta inicial}

Por medio del examen físico (que incluye el tacto rectal) y radiografía de abdomen simple, se evalúa la presencia de masas fecales, distensión abdominal, dermatitis perianal, calibre y altura de la anastomosis. En los pacientes con impactación fecal, se indican enemas oleosalinas con vaselina (1/3) y solución fisiológica (2/3) en forma secuencial, con sonda rectal. Volumen: de 10 a $20 \mathrm{ml} / \mathrm{kg}$, 1 o 2 por día. Una alternativa es la desimpactación por vía oral con polietilenglicol durante 4-6 días.

En los pacientes constipados sin impactación, se indican laxantes osmóticos. El polietilenglicol es el más efectivo, bien tolerado y seguro. ${ }^{17,18}$ Algunos pacientes pueden beneficiarse con lactulosa. Cuando no hay respuesta, se agregan laxantes estimulantes como bisacodilo. El dolor abdominal, efecto adverso frecuente, habitualmente, mejora con la reducción de la dosis ${ }^{19}$ (Figura 1 y Tabla 1 ).

En los pacientes con síntomas crónicos, se recomienda mantener el tratamiento, al menos, por dos meses luego de haberse resuelto los síntomas sin interrumpirlo precozmente. Después de este período, se debe descender gradualmente la dosis en caso de buena respuesta. Si recaen o persisten sintomáticos, se inicia el algoritmo diagnóstico-terapéutico de síntomas obstructivos (Figura 2).

\section{INCONTINENCIA}

Para algunos autores, la incontinencia es la complicación más frecuente (en el $50 \%$ ) (6,1 $^{1}$ y la de más difícil manejo. La continencia requiere sensorialidad normal del canal anal, adecuado control de esfínteres y motilidad colónica conservada. ${ }^{20}$ La disrupción de alguno de estos factores convierte al paciente en total o parcialmente incontinente. ${ }^{21,22}$

¿Por qué es tan frecuente la incontinencia posquirúrgica si los pacientes nacen con su mecanismo de continencia intacto y las distintas técnicas quirúrgicas tratan de preservarla? 


\section{Causas}

1. Compromiso esfinteriano durante el descenso o como resultado de miectomías o esfinterotomías previas. Las técnicas que requieren gran disección (Swenson o Soave),

TABLA 1. Laxantes en pediatría

\begin{tabular}{|c|c|c|}
\hline Laxantes & Dosis & Efecto \\
\hline Lactulosa & 1-3 ml/kg/día. & Efecto osmótico. \\
\hline $\begin{array}{l}\text { Hidróxido } \\
\text { de magnesio }\end{array}$ & $\begin{array}{l}\text { 1-3 ml } / \mathrm{kg} / \text { día } \\
\text { de una solución } \\
\text { de } 400 \mathrm{mg} / 5 \mathrm{ml} .\end{array}$ & $\begin{array}{c}\text { Efecto } \\
\text { osmótico. }\end{array}$ \\
\hline $\begin{array}{l}\text { Aceite } \\
\text { mineral }\end{array}$ & $\begin{array}{l}\text { Desimpactación: } \\
\text { 15-30 } \mathrm{ml} / \text { año de edad } \\
\text { hasta } 240 \mathrm{ml} / \text { día. } \\
\text { Mantenimiento: } \\
\text { 1-3 } \mathrm{ml} / \mathrm{kg} \text { / día. }\end{array}$ & $\begin{array}{c}\text { Efecto } \\
\text { lubricante. } \\
\text { < de } 1 \text { año: } \\
\text { no recomendado. } \\
\text { Riesgo de neumonía } \\
\text { lipoidea por } \\
\text { aspiración. }\end{array}$ \\
\hline $\begin{array}{l}\text { Polietilen- } \\
\text { glicol } \\
3350\end{array}$ & $\begin{array}{c}\text { Desimpactación: } \\
\text { 1-1,5 g/ kg/día/3 días. } \\
\text { Mantenimiento: } \\
\text { 0,8 g/ kg/día. }\end{array}$ & $\begin{array}{c}\text { Efecto } \\
\text { osmótico. }\end{array}$ \\
\hline Bisacodilo & $\begin{array}{l}\text { 3-12 años: } 5 \text { mg/ día. } \\
\text { > } 12 \text { años y adultos: } \\
\text { 5-15 mg/ día cada } 24 \text { h. } \\
\text { Comprimidos de } 5 \text { mg. }\end{array}$ & $\begin{array}{c}\text { Efecto } \\
\text { estimulante. }\end{array}$ \\
\hline $\begin{array}{l}\text { Picosulfato } \\
\text { de sodio }\end{array}$ & $\begin{array}{c}\text { < } 4 \text { años: 0,25 mg/kg/día. } \\
\text { 4-10 años: 2,5-5 mg/día. } \\
\text { > } 10 \text { años: 5-10 mg/ día. } \\
\text { Gotas de 7,5 mg/ml. }\end{array}$ & $\begin{array}{l}\text { Efecto } \\
\text { estimulante. }\end{array}$ \\
\hline
\end{tabular}

Fuente propia. el abordaje transanal exclusivo (De la Torre) o una anastomosis a nivel o debajo de la línea pectínea aumentan este riesgo. Se sospecha, al observar la interrupción de la línea pectínea, un ano entreabierto, prolapso de la mucosa o grave dermatitis papuloerosiva. Se confirma con manometría anorrectal que muestra esfínteres hipotensivos y ecografía endoanal.

2. Alteraciones de la sensorialidad: en caso de una anastomosis muy baja, más frecuente con las técnicas de Swenson o Soave y menos luego de la cirugía de Duhamel.

3. Algunos pacientes pueden experimentar escurrimiento por rebosamiento (se deben investigar síntomas obstructivos).

4. El exceso de azúcares en la dieta es causa de incontinencia, urgencia defecatoria y elevada frecuencia de deposiciones.

5. Hipermotilidad del colon proximal: frecuente después de la cirugía, aun durante el sueño. Explica la incontinencia diurna y, sobre todo, nocturna. ${ }^{6,23}$

6. Alteraciones del neurodesarrollo: los niños mayores de 4 años pueden referir ausencia de sensación defecatoria sin otras alteraciones anatómicas o funcionales que la justifiquen. Obedece a la falta de reconocimiento de las señales aferentes, lo cual se modifica a medida que el niño adquiere el hábito evacuatorio adecuado. ${ }^{6}$

\section{Tratamiento}

Dieta hipofermentativa, anticolinérgicos (loperamida), agentes formadores de masa, como psyllium o pectinas, y biofeedback para mejorar la contracción voluntaria. Para la
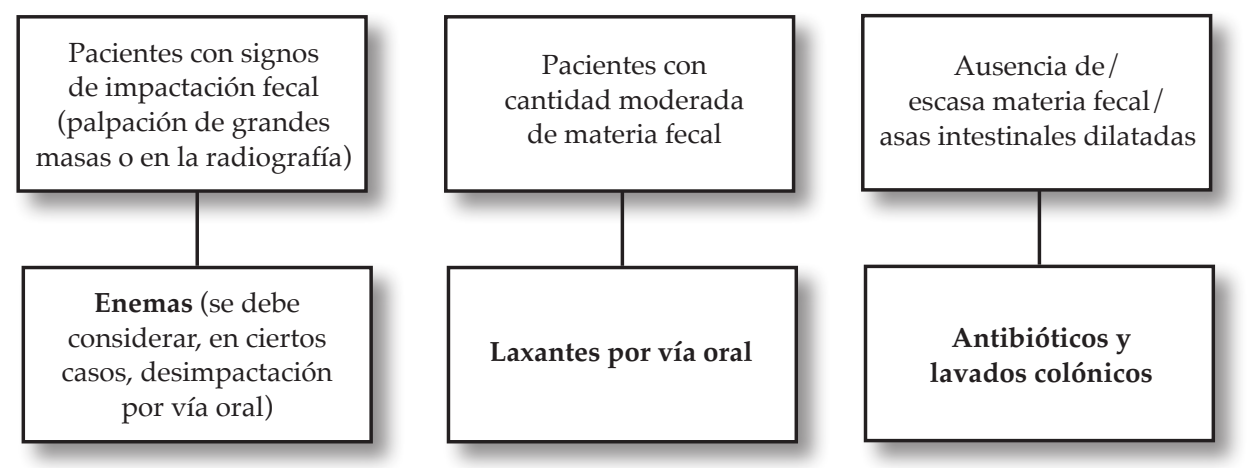

Tabla adaptada de Hoekman D, Benninga MA. Functional constipation in childhood: current pharmacotherapy and future perspectives. Expert Opin. Pharmacother. 2013;14:41-51. 
incontinencia nocturna, se ha propuesto el uso de antidepresivos tricíclicos, como amitriptilina nocturna. Por su efecto anticolinérgico, reduce la presión de las ondas. ${ }^{6}$

Cuando existe compromiso esfinteriano grave, pueden agregarse enemas de poco volumen o considerar ostomías. Recientemente, se han incorporado las irrigaciones transanales como alternativa útil en los pacientes con disfunción intestinal (incontinencia o constipación refractaria) que no responden a otros tratamientos ${ }^{24}$ (Tabla 2).

\section{ENTEROCOLITIS}

Su incidencia varía entre el $6 \%$ y el $26 \%$ en el período preoperatorio o al momento del diagnóstico y del $5 \%$ al $42 \%$ luego de la cirugía correctora de la EH. ${ }^{25-29}$ Los episodios son más frecuentes en los primeros dos años posteriores a la cirugía. Los factores de riesgo para el desarrollo de enterocolitis son el síndrome de Down y la enfermedad de segmento largo o total. ${ }^{29,30}$

La patogenia no es clara: el SNE inmaduro del intestino remanente podría crear un equilibrio anormal que puede romperse por una obstrucción

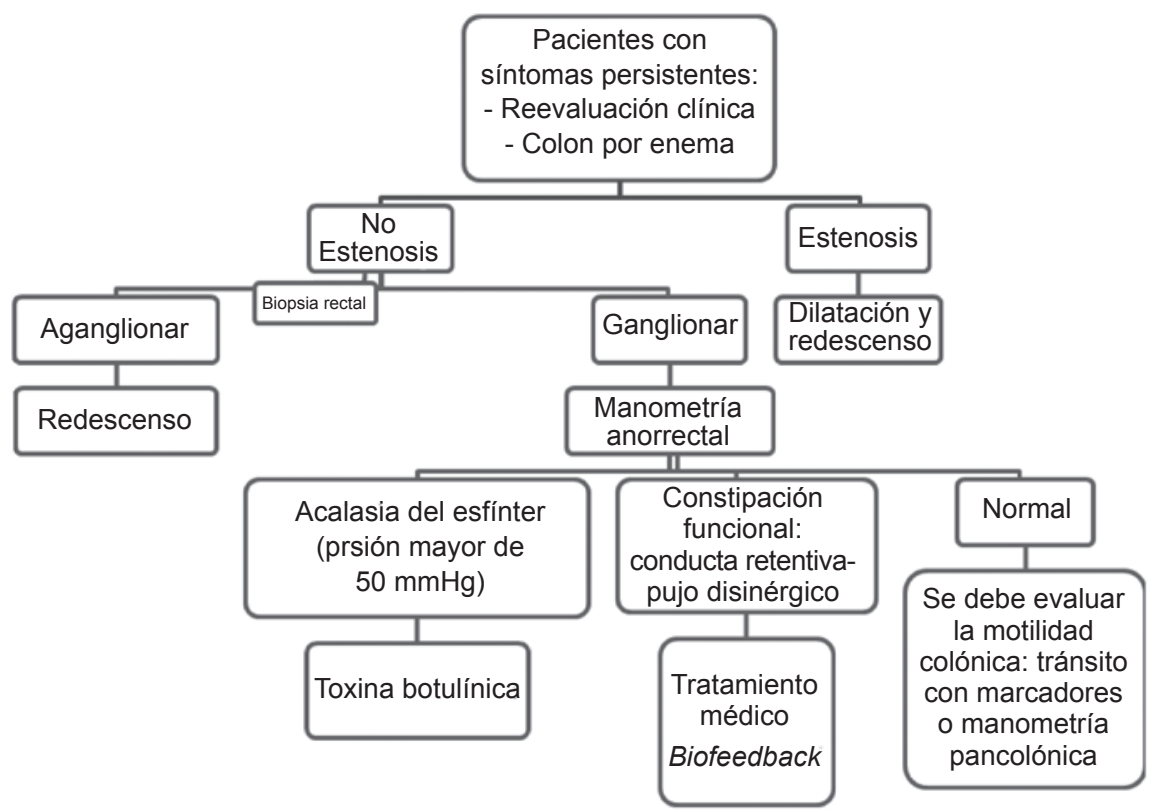

Figura adaptada de Langer JC, Rollins MD, Levitt M, Gosain A, et al; American Pediatric Surgical Association Hirschsprung Disease Interest Group. Guidelines for the management of postoperative obstructive symptoms in children with Hirschsprung disease. Pediatric Surg Int. 2017;33:523-526.

TABLA 2. Incontinencia: causas, cómo estudiarla y tratamiento

\begin{tabular}{lcc}
\hline Causas & Estudio & Tratamiento \\
\hline $\begin{array}{l}\text { Alteración de la función } \\
\text { de los esfínteres o de la sensorialidad. }\end{array}$ & Examen físico, & Anticolinérgicos (loperamida). \\
& se debe evaluar la línea pectínea. & Formadores de masa (psyllium). \\
Hipermotilidad en el colon proximal. & Manometría anorrectal. & Biofeedback. \\
Excesos de azúcares en la dieta. & Manometría pancolónica. & \\
Escurrimiento por rebosamiento. & Evaluación individualizada & Dieta hipofermentativa. \\
\hline
\end{tabular}

Tabla adaptada de Mosiello G1, Marshall D, Rolle U, Crétolle C, et al. Consensus review of best practice of transanal irrigation in children. J Pediatr Gastroenterol Nutr. 2017 Mar;64(3):343-352. 
parcial o el sobredesarrollo bacteriano y ocasionar enterocolitis. ${ }^{31}$ Un estudio reciente demostró que los pacientes con enterocolitis tienen un microbioma considerablemente diferente al de los individuos sanos, con una pérdida de la diversidad y expansión patológica de bacterias. ${ }^{32}$

El cuadro clínico es variable. Las formas leves y moderadas se caracterizan por deposiciones líquidas, fétidas y frecuentes. Ocasionalmente, hay ausencia de catarsis, con importante distensión abdominal y, al colocar una sonda o realizar tacto rectal, se obtiene una deposición explosiva. Puede haber rechazo del alimento, vómitos, irritabilidad $\mathrm{y}$, en las formas más graves, fiebre, letargo, deposiciones con sangre, deshidratación y shock, que configuran un megacolon tóxico. ${ }^{30}$

El diagnóstico es clínico. En la radiografía de abdomen, se observan asas dilatadas, niveles hidroaéreos, aspecto dentado del borde intestinal, nivel en el rectosigma sin aire distal y neumatosis. Estos hallazgos pueden confundirse con oclusión mecánica y llevar a laparotomías innecesarias. El colon por enema y la colonoscopía están contraindicados por el riesgo de perforación. El tratamiento consiste en antibióticos, descompresión y lavados colónicos.

Formas de leves a moderadas: son de manejo a mbulatorio con antibióticos por vía oral (metronidazol de elección). Las formas graves requieren antibióticos por vía parenteral.

Lavados colónicos: deben hacerse con sonda gruesa y solución fisiológica tibia a razón de $10-20 \mathrm{ml} / \mathrm{kg}$ de 2 a 4 veces por día. El líquido infundido por la sonda debe aspirarse y lavar hasta que salga claro. En los casos graves, están contraindicados por el riesgo de perforación, pero puede intentarse la descompresión con la colocación cuidadosa de una sonda rectal. Ante una inadecuada descompresión colónica, sepsis o desmejoramiento del estado general, deberá considerarse una ostomía (Tabla 3).

En caso de enterocolitis recurrente, se indican ciclos de cinco días de antibióticos mensuales (metronidazol o amoxicilina clavulánico). ${ }^{10}$ Los pacientes con megacolon total presentan los episodios más frecuentes y graves, y requieren tratamientos prolongados para mejorar la calidad de las deposiciones y evitar recaídas. Una revisión sistemática sobre el uso de probióticos no demostró beneficios. ${ }^{33}$

En casos de enterocolitis recurrente: 1) Se debe revisar la pieza quirúrgica para ver si se ha descendido intestino aganglionar, de transición o con displasia neuronal. 2) Se debe realizar colon por enema para evaluar alteraciones anatómicas y biopsia rectal por succión para descartar segmento de aganglionosis residual. 3) Se debe realizar manometría anorrectal para buscar acalasia del esfínter y considerar la toxina botulínica en aquellos con presiones elevadas de esfínter anal interno..$^{30} 4$ ) Si no hay una obstrucción funcional del tracto de salida, se debe evaluar la motilidad del colon o del intestino delgado remanente con manometría pancolónica.

\section{TRATAMIENTO NUTRICIONAL}

La dietoterapia busca mantener un adecuado estado nutricional y se modifica durante las etapas pre- y posquirúrgicas. En los pacientes con ostomía, su localización tiene impacto en la absorción y en el estado nutricional. En los ileostomizados, debe adaptarse el plan alimentario para disminuir las pérdidas, asegurar una adecuada absorción de nutrientes y aporte calórico, y es habitual en los lactantes y los niños pequeños el soporte nutricional con fórmula sin lactosa o hidrolizada por vía oral o por sonda.

Luego del descenso, el objetivo es evitar la diarrea, el deterioro del estado nutricional y la dermatitis grave en la zona de pañal. Esta produce irritabilidad, limita la ingesta, altera el sueño y la movilidad por dolor, lo que perturba la calidad de vida.

Aun los pacientes con resección de segmento corto pueden presentar diarrea en los primeros meses poscirugía. Inicialmente, las deposiciones son 5-10 por día y descienden a 1-4 al día entre los 6 y los 12 meses posteriores. ${ }^{34}$

En los pacientes con colectomía total sin válvula ileocecal, la resección del íleon terminal afecta la absorción de agua, electrolitos, vitamina B12 y folato. ${ }^{35,36}$ Las deposiciones son más frecuentes, líquidas o desligadas.

\section{TABLA 3. Tratamiento: enterocolitis}

Descompresión o lavados colónicos

- Solución fisiológica tibia 10-20 ml/ kg.

- Sonda gruesa.

- Con rescate.

\section{Antibióticos}

- Casos de leves a moderados: metronidazol por vía oral, 10/15 días.

- Casos graves: por vía endovenosa, de amplio espectro.

Falta de respuesta, sepsis, empeoramiento

- Se debe considerar la ostomía.

Fuente propia. 
La diarrea y la dermatitis perianal son más graves en los lactantes y los niños pequeños que reciben leche de madre y/o consumen fórmulas lácteas comunes. Estos pacientes sobrellevan mejor el período posquirúrgico inmediato, cuando se restringen o limitan los disacáridos de la dieta, con el uso de una fórmula sin lactosa y una dieta hipofermentativa con muy bajo contenido en lactosa, sacarosa y mínima o nula cantidad de fibra soluble (Tabla 4. A).

Cuando no se logra la adecuada ganancia de peso o no se cubren los requerimientos, se debe considerar el soporte nutricional enteral. La progresión de la dieta es individual según la resección intestinal y la tolerancia, y se adaptan las necesidades y los gustos, por lo que es necesario el asesoramiento de un licenciado en Nutrición.

\section{Incontinencia}

Los pacientes que tienen un colon hiperreactivo, con tendencia a la hipermotilidad y diarrea, se benefician con una dieta hipofermentativa $y$, en algunos casos, el agregado de pectinas (fibra soluble) con poco líquido para lograr el aumento de volumen y mejoría de la consistencia de las deposiciones. ${ }^{37}$ La fibra soluble tiende a dar consistencia a la materia fecal, mientras que la fibra insoluble tiende a desligarla. ${ }^{38}$

\section{Constipación}

Para el tratamiento de la constipación funcional, las guías de la European Society for Paediatric Gastroenterology, Hepatology and Nutrition (ESPGHAN) y la North American Society for Pediatric Gastroenterology, Hepatology, and Nutrition (NASPGHAN) recomiendan un consumo normal de fibra y adecuada ingesta de líquido. ${ }^{18}$ Un enfoque racional para menores de 2 años es introducir variedad de vegetales, frutas y cereales de fácil digestión a medida que se incorporan alimentos en la dieta. La recomendación para los niños mayores es "5 g/ día + edad actual" hasta alcanzar 25-35 g/ día. En los pacientes con EH con constipación, se

\title{
TABLA 4. Recomendaciones dietoterápicas
}

\section{A. Recomendaciones dietoterápicas ante la diarrea}

Se debe limitar la lactosa:

- Limitar el consumo de leche común o fórmulas lácteas con lactosa.

- Reemplazar por una fórmula sin lactosa o leche reducida en lactosa, según la edad y las características de las deposiciones.

- Evaluar la necesidad de complementar con una fórmula sin lactosa y / o hidrolizada, según la condición clínica en los lactantes amamantados, en caso de diarrea y/o mal progreso de peso.

- Considerar el uso de una fórmula hidrolizada en los pacientes colectomizados.

Se debe limitar la sacarosa:

- Evitar los alimentos ricos en azúcares simples (mermeladas, jaleas, golosinas, galletitas, budines, helados y productos de pastelería dulces).

- Evitar jugos de frutas y bebidas azucaradas con agregado de sacarosa, fructosa, jarabe de maíz de alta fructosa (JMAF), incluso los jugos naturales.

\section{Fibra soluble:}

- Se debe seleccionar y / o limitar el consumo de frutas y vegetales cocidos / pelados.

- Se deben consumir con moderación alimentos ricos en fibra soluble (avena arrollada, calabaza, zanahoria, banana madura, manzana cocida sin cáscara, etc.) según la tolerancia.

\section{B. Recomendaciones dietoterápicas ante la constipación}

\author{
Alimentos ricos en fibra: \\ Se evaluará la tolerancia individual (cocidos-crudos, cantidad y variedad según el aporte de fibra) \\ - Consumir variedad de frutas, vegetales. \\ - Incorporar avena y salvado de avena en las preparaciones. \\ - Preferir cereales de grano entero integrales (ej.: arroz integral). \\ - Preferir panificados integrales y / o con salvado de trigo y copos de cereales (sin azúcar). \\ - Consumir legumbres (lentejas, arvejas, porotos, garbanzos).
}


aconseja aumentar en forma progresiva la ingesta de fibra, evaluando la tolerancia individual. Se debe iniciar con alimentos que contengan fibra de predominio soluble $y$, según la tolerancia, incorporar luego alimentos con fibra insoluble, asegurando el consumo suficiente de líquido (Tabla 4. B). ${ }^{35-40}$

Deberá considerarse también que, entre los 2 y los 4 años, hay una incidencia mayor de estreñimiento coincidente con la etapa de entrenamiento para ir al baño. Es fundamental crear el hábito evacuatorio, mantener una rutina de reeducación de esfínteres, establecer momentos para defecar, considerando horarios y actividades (20-40 minutos poscomida y antes del sueño para evitar escapes nocturnos). ${ }^{41}$

\section{CONCLUSIONES}

La cirugía correctora de la EH no siempre logra un hábito defecatorio y una continencia normales. El compromiso del SNE no está limitado al segmento aganglionar, lo que explica, en parte, que estos pacientes presenten síntomas en forma persistente. La etiología es multifactorial, por lo que es importante implementar un algoritmo diagnóstico-terapéutico que pueda realizarse en nuestro medio. La complejidad hace que estos niños se beneficien con un seguimiento multidisciplinario periódico.

\section{REFERENCIAS}

1. Heukeroth R. Hirschsprung disease. In: Faure C, Di Lorenzo C, Thapar N (eds.). Pediatric Neurogastroenterology: Gastrointestinal Motility and Functional Disorders in Children. $2^{\text {nd }}$ ed. Cham: Springer; 2017.Págs.291-302.

2. Swenson O, Bill AH. Resection of the rectum and rectosigmoid with preservation of the sphincter for benign spastic lesions producing megacolon. Surgery. 1948; 24(2):212-20.

3. Soave F. A new surgical technique for treatment of Hirschsprung's disease. Surgery. 1964; 56:1007-14.

4. Duhamel B. A new operation for the treatment of Hirschsprung's disease. Arch Dis Child. 1960; 35:38-9.

5. Georgeson KE, Cohen RD, Hebra A, Jona DM, et al. Primary laparoscopic-assisted endorectal colon pull-through for Hirschsprung's disease: A new gold standard. Ann Surg. 1999; 229(5):678-82.

6. Hyman P. Defecation disorders after surgery for Hirschsprung's disease. J Pediatr Gastroenterol Nutr. 2005; 41(Suppl 1):S62-3.

7. Calkins C. Hirschsprung disease beyond infancy. Clin Colon Rectal Surg. 2018; 31(2):51-60.

8. Jarvi K, Laitakari EM, Koivusalo A, Rintala RJ, et al. Bowel function and gastrointestinal quality of life among adults operated for Hirschsprung disease during childhood: a population-based study. Ann Surg. 2010; 252(6):977-81.

9. Langer JC, Rollins MD, Levitt M, Gosain A, etal. Guidelines for the management of postoperative obstructive symptoms in children with Hirschsprung disease. Pediatric Surg Int. 2017; 33(5):523-6.
10. Levitt M, Dickie B, Peña A. The Hirschsprungs patient who is soiling after what was considered a "successful" pullthrough. Semin Pediatr Surg. 2012; 21(4):344-53.

11. Chumpitazi B, Nurko S. Defecation Disorders in children after surgery for Hirschsprung disease.JPediatr Gastroenterol Nutr. 2011; 53(1):75-9.

12. Keshtgar A, Choudhry MS, Kufeji D, Ward HC, et al. Anorectal manometry with and without ketamine for evaluation of defecation disorders in children. J Pediatr Surg. 2015; 50(3):438-43.

13. Rodríguez L, Sood M, Di Lorenzo C, Saps M. An ANMSNASPGHAN consensus document on anorectal and colonic manometry in children. Neurogastroenterol Motil. 2017; 29(1):e12944

14. Madrid SA. Revisión crítica de los métodos utilizados en el estudio del tránsito colónico. Gastroenterol Latinoam. 2008; 19(2):81-5.

15. Ciamarra P, NurkoS, Barksdale E, Fishman S, et al. Internal anal sphincter achalasia in children: clinical characteristics and treatment with Clostridium botulinum toxin. J Pediatr Gastroenterol Nutr. 2003; 37(3):315-9.

16. Wester T, Granstrom AL. Botulinum toxin is efficient to treat obstructive symptoms in children with Hirschsprung disease. Pediatr Surg Int. 2015; 31(3):255-9.

17. Gordon M, Naidoo K, Akobeng AK, Thomas AG. Cochrane Review: Osmotic and stimulant laxatives for the management of childhood constipation. Evid Based Child Health. 2013; 8(1):57-109.

18. Tabbers MM, Di Lorenzo C, Berger MY, Faure C, et al. Evaluation and treatment of functional constipation in infants and children: Evidence-based recommendations from ESPGHAN and NASPGHAN. J Pediatr Gastroenterol Nutr. 2014; 58(2):258-74.

19. Hoekman D, Benninga MA. Functional constipation in childhood: current pharmacotherapy and future perspectives. Expert Opin Pharmacother. 2013; 14(1):41-51.

20. LevittMA, Peña A. Update on pediatric faecal incontinence. Eur J Pediatr Surg. 2009; 19(1):1-9.

21. LevittMA, Peña A. Pediatricfecal incontinence: A surgeon's perspective. Pediatr Rev. 2010; 31(3):91-101.

22. De La Torre L, Wehrli LA. Error traps and Culture of Safety in Hirschsprung disease. Semin Pediatr Surg. 2019; 28(3):151-9.

23. Kaul A, Garza JM, Connor FL, Cocjin JT, et al. Colonic hyperactivity results in frequent fecal soiling in a subset of children after surgery for Hirschsprung disease. J Pediatr Gastroenterol Nutr. 2011; 52(4):433-6.

24. Mosiello G, MarshallD, RolleU, CrétolleC, et al. Consensus review of best practice of transanal irrigation in children. J Pediatr Gastroenterol Nutr. 2017; 64(3):343-52.

25. Elhalaby EA, Coran AG, Blane CE, Hirschl RB, et al. Enterocolitis associated with Hirschsprung's disease: a clinical-radiological characterization based on 168 patients. J Pediatr Surg. 1995; 30(1):76-83.

26. Imamura A, Puri P, O'Briain DS, Reen DJ. Mucosal immune defence mechanisms in enterocolitis complicating Hirschsprung's disease. Gut. 1992; 33(6):801-6.

27. Pastor AC, Osman F, Teitelbaum DH, Caty MG, et al. Development of a standardized definition for Hirschsprung's-associated enterocolitis: a Delphi analysis. J Pediatr Surg. 2009; 44(1):251-6.

28. Rescorla F, Morrison A, Engles D, West KW, et al. Hirschsprung's disease. Evaluation of mortality and longterm function in 260 cases. Arch Surg. 1992; 127(8):934-41.

29. Teitelbaum D, Qualman S, Caniano D. Hirschsprung's disease. Identification of risk factors for enterocolitis. Ann Surg. 1988; 207(3):240-4.

30. Gosain A, Frykman PK, Cowles RA, Horton J, et al. 
Guidelines for the diagnosis and management of Hirschsprung-associated enterocolitis. Pediatr Surg Int. 2017; 33(5):517-21.

30. Romo Muñoz MI, Martínez de Aragón A, Núñez Cerezo V, Udaondo C, et al. Factores de riesgo asociados al desarrollo de enterocolitis en la enfermedad de Hirschsprung. Cir Pediatr. 2018; 31(1):34-8.

31. Demehri F, Halaweish I, Coran A, Teitelbaum D. Hirschsprung-associated enterocolitis: pathogenesis, treatment and prevention. Pediatr Surg Int. 2013; 29(9):873-81.

32. Neuvonen MI, Korpela K, Kyrklund K, Salonen A, et al. Intestinal microbiota in Hirschsprung disease. J Pediatr Gastroenterol Nutr. 2018; 67(5):594-600.

33. Nakamura H, Lim T, Puri P. Probiotics for the prevention of Hirschsprung associated enterocolitis: a systematic review and meta-analysis. Pediatr Surg Int. 2018; 34(2):189-93.

34. Haricharan RN, Georgeson KE. Hirschsprung disease. Semin Pediatr Surg. 2008; 17(4):266-75.

35. Nevin-Folino NL, American Dietetic Association. Pediatric
Nutrition Practice Group. Pediatric Manual of Clinical Dietetics. $2^{\text {nd }}$ ed. Chicago: American Dietetic Association; 2008.

36. Blesa Sánchez E, Enríquez Zarabozo E, Marín Santander D, Ayuso Velasco R, et al. Repercusión de distintos tipos de colectomía sobre el estado de nutrición y tipo de heces. Cir Pediatr. 2009; 22(3):145-9.

37. RintalaRJ,PakarinenMP.Long-termoutcomes ofHirschprung s disease. Semin Pediatr Surg. 2012; 21(4):336-43.

38. Axelrod $\mathrm{CH}$, Saps $\mathrm{M}$. The role of fiber in the treatment of functional gastrointestinal disorders in children. Nutrients. 2018; 10(11):E1650.

39. Korczak R, Kamil A, Fleige L, Donovan S, etal. Dietary fiber and digestive health in children. Nutr Rev. 2017; 75:241-59.

40. DahlWJ, Stewart ML. Position of the Academy of Nutrition and Dietetics: Health Implications of Dietary Fiber. J Acad Nutr Diet. 2015; 115(11):1861-70.

41. Bischoff A, Levitt M, Peña A. Bowel management for the treatment of pediatric fecal incontinence. Pediatr Surg Int. 2009; 25(12):1027-42. 